\title{
Specific drugs for a complex disease: can there be a magic bullet against rheumatoid arthritis?
}

\author{
Hans Richard Barthel, Gerd R Burmester
}

Advanced rheumatoid arthritis (RA) represents a complex disease, characterised by disturbances in a number of different biological pathways. The current most effective treatments, such as gold, methotrexate, and steroids act rather non-selectively and simultaneously on various biological pathways, thus presumably achieving their benefit by a complex interplay with different biological networks. Various side effects are the logical consequences. In most of the current literature there is a quest for more specific treatments that would avoid the side effects of currently available medications. This article questions whether highly selective drugs, including biological agents targeting precise points in complex networks, can be superior to medications with a broader spectrum of action. Experience with other complex diseases, such as hypertension, has taught us that single drug regimens are rarely successful in the control of advanced disease.

What is our understanding of RA today? Despite an intensive search for an infectious agent in RA, none has yet been unequivocally identified; Lyme arthritis reminds us that such an agent can remain elusive for a long time. Nevertheless, evidence is evolving that RA is a two stage disease. Very early in its course, $T$ cells seem to be important-a hypothesis supported first, by the association of RA with certain major histocompatibility complex class II antigens that share a common amino acid sequence located in their peptide binding groove, ${ }^{1}$ and second, by the finding of a clonally restricted $T$ cell proliferation when $V \beta$ chains of the synovial $T$ cell receptors are analysed in patients with early disease. ${ }^{2}$ However, overall, other investigations have failed to identify unequivocally such a V $\beta$ family. ${ }^{3}$ In advanced $\mathrm{RA}, \mathrm{T}$ cells seem not to be the major players. Macrophages and monocytes become more important, as they contribute the majority of proinflammatory cytokines, ${ }^{4}$ while $T$ cell specific cytokines are scarcely detectable. In addition, fibroblast-like synoviocytes (type 2 synoviocytes) are found to invade and erode cartilage. These cells grow independently of stimuli from other cells in culture, thus demonstrating tumour-like growth, at least for a certain period. ${ }^{5}$ It is not yet clear whether the two stages described occur sequentially in time or if, in later phases of the disease, a continuing $T$ cell drive is still important, but difficult to detect. Homing of inflammatory cells, directed by adhesion molecules and chemoattractants, helps further to localise the inflammatory process at the synovium.
In addition to its inflammatory features, RA is characterised by disturbances of the hormonal and neuroendocrine network. ${ }^{6}$ Whether these disturbances are secondary phenomena or represent an initiating event, remains unclear.

\section{The problems of intervening in the complex processes of $R A$}

According to the model described above, very early intervention would reduce the problems of complexity encountered increasingly in late stage disease. In particular, treatments directed against the presentation of (auto?) antigens to $T$ cells should be advantageous early in the disease. However, in contrast to results with animals, a recent trial of monoclonal antibodies to $T$ cells in early RA failed to substantiate this hypothesis. ${ }^{7}$ It may be, therefore, that early disease in humans already reflects widespread disturbances to biological networks that, in comparison with early disease stages in animals, are too far advanced to permit such a specifically targeted intervention to be effective, especially as our current strategies may not be sufficiently radical to deplete an effective number of $T$ cells. However, the failure of one approach directed against $T$ cells certainly does not preclude other strategies being effective: non-depleting $T$ cell antibodies, and blocking agents that would prevent lymphocyte trafficking, are under investigation. Cyclosporin shows some effect in late stage disease, and may be even more effective in early disease, but has not yet been fully investigated in this respect.

In advanced RA, the complexity of the biological pathways involved becomes even more relevant. Treatment should target the monocyte-macrophage system, as it is the major source of proinflammatory cytokines. However, a vast array of different monokines are expressed and it remains unclear if there is a chance to downregulate or reverse the monocyte-macrophage activation status as a whole for a prolonged period of time. Some cytokines might exert positive feedback, as has been suggested for tumour necrosis factor $\alpha$ $(\mathrm{TNF} \alpha)$. However, though studies of treatment with anti-TNF $\alpha$ showed that symptoms were improved, preliminary data have so far failed to support a general downregulatory effect on other cytokines. ${ }^{8}$ Interventions would be particularly effective if there were a hierarchy of cytokines, with one upregulating the others. There is evidence for the existence of such triggers. Circulatory monocytes are activated in RA patients. However, re- 
populating monocytes are not activated initially, but become activated later in their life span. ${ }^{9}$ Similarly, relapse of RA has been reported as late as three to four years after bone marrow transplantation for concomitant disease. ${ }^{1011}$ Therefore, it would make much more sense to counteract such activating triggers, rather than blocking 'downstream' cytokines or a self perpetuating network. However, despite intensive research into the activation of the monocyte-macrophage system in $\mathrm{RA}$, the nature of the triggers leading to these events is not clear.

It is possible, also, that clinical studies might fail to demonstrate superior efficacy of a treatment because of the diversity of causative factors. What is called RA most probably represents a spectrum of different diseases caused by different mechanisms; this may be particularly pertinent to the seronegative variety of the disorder. Reports of an average response in statistically evaluated studies may therefore miss a meaningful response in certain subsets of patients that may be overlooked when evaluating the patient population as a whole. Whether this diversity of causative factors has important implications for treatment may be answered once we are able to compare cytokine profiles in individual patients, especially with regard to certain HLA backgrounds.

The cytokine network is extremely complex and far from being understood. Therapeutic interventions in this network are much more difficult to understand than interventions in the endocrine system network. Today's knowledge is only crude, as we often describe the interaction of just two cytokines in our experiments. How can we describe a system of 15 cytokines (or may be more?) that all influence each other, especially in vivo? Mathematical models have been successfully applied to problems in the field of immunology; ${ }^{12}$ such an approach might become a useful tool in the increasingly complex field of immunological research, notably in the study of cytokines.

\section{Highly specific versus combined treatments?}

Currently, highly selective treatments such as monoclonal antibodies directed against cell surface constituents, fusion toxins against cell activation markers, cytokines and cytokine inhibitors are under investigation for the treatment of RA. ${ }^{13}$ However, most of the results reported to date have been from open trials (phase 1 studies). Controlled studies with antiTNF $\alpha$ are promising, ${ }^{14}$ while controlled trials evaluating anti-CD $4,{ }^{7}$ oral tolerisation, ${ }^{15}$ and interferon gamma ${ }^{16}{ }^{17}$ had difficulty demonstrating a relevant benefit. Furthermore, these medications still await comparison with standard treatments such as intramuscular gold or methotrexate (MTX). Although all specific drugs are highly effective in animal models, transfer to humans has had no or only partial success. This discrepancy has not been fully elucidated, but suggests either that RA is too complex to be targeted effectively by a single specific agent, or that we have not yet identified the appropriate target.

Whether combinations of specific drugs are advantageous over 'broad spectrum' drugs such as we are using now remains unanswered, but will fuel research in forthcoming years. As combining many highly specific drugs is an option not yet available, combined treatments using conventional drugs are currently being studied intensively, encouraged by new results. Combination of MTX with sulphasalazine has been shown to be superior to MTX alone, ${ }^{18}$ and the addition of cyclosporin improved their disease in at least a significant number of patients with primary or secondary failure of MTX treatment. ${ }^{19}$ Moreover, new data have revealed triple treatment (MTX-sulphasalazine-hydroxychloroquine) to be particularly promising. ${ }^{20}$ With respect to specificity, combinations of conventional drugs represent the other extreme compared with highly selective biological agents. The fact that one of the most effective treatment strategies comprises combining drugs that each have unknown therapeutic actions when used alone, reflects how difficult-if not impossible - it currently is to target the available treatments towards specific inflammatory mechanisms.

\section{Summary}

The complexity of RA challenges our search for a better understanding of the interconnected networks. Early treatment will certainly avoid some of the problems presented by the complexity of the disease, and combined treatments seem advantageous for advanced disease. The complexity of overt RA will probably not allow a single agent to exert superior efficacy. Unless a treatable infectious cause is identified, a magic bullet, though theoretically possible, might not exist in reality, in particular if the disease is advanced.

1 Gregerson P K, Silver J, Winchester R J. The shared epitope hypothesis. An approach to understanding the molecular genetics of susceptibility to rheumatoid arthritis. Arthritis Rheum 1987; 30: 1205-13.

2 Bucht A, Oksenbreg J R, Lindblat S, Grönberg A Steinmann L, Klareskog L. Characterization of T-cell receptor alpha beta repertoire in synovial tissue from different temporal phases of rheumatoid arthritis. Scand f Immunol 1992; 35: 159-65.

3 Zagon G, Tumang J R, Li Y, Friedman S M, Crow M K Increased frequency of $\mathrm{V} \beta 17$-positive $\mathrm{T}$ cells in patients with rheumatoid arthritis. Arthritis Rheum 1994; 37: 1431-40.

4 Firestein G S, Alvaro-Garcia J M, Maki R. Quantitative analysis of cytokine expression in rheumatoid arthritis. f Immunol 1990; 144: 3347-53.

5 Tsai V, Alsalameh S, Boyle D, Firestein G, Zwaifler N. Pannocytes: distinctive cells derived from sites of articular Cartilage injury in rheumatoid arthr

6 Jorgensen C, Sany J. Modulation of the immune response by the neuro-endocrine axis in rheumatoid arthritis. Clin Exp Rheumatol 1994; 12: 435-41.

7 Van der Lubbe P A, Dijkmans B A C, Markusse H M, Nässander U, Breedwald F L. A randomized, doubleblind, placebo-controlled study of CD4 monoclonal antibody therapy in early rheumatoid arthritis. Arthritis Rheum 1995; 38: 1097-110.

8 Lorenz H M, Antoni C, Geiler T, Schwerdtner N, Kalden J R, Manger B. IV infusion of a TNF- $\alpha$ antibody in patients with rheumatoid arthritis: changes on the cellular and molecular level (abstract). Z Rheum 1994; 53(suppl 1): 30 .

9 Hahn G, Stuhlmüller B, Hain R, Kalden J R, Pfizenmaier K, Burmester G R. Modulation of monocyte activation in patients with rheumatoid arthritis by leukapheresis therapy. $\mathcal{f}$ Clin Invest 1993; 91: 862-70. 
10 Jacobs P, Vincent M D, Martell R W. Prolonged remission of severe refractory theumatoid arthritis following allogenic bone marrow transplantation for drug-induced aplastic anemia. Bone Marrow Transplant 1986; 1: 237-339.

11 McKendry R J R, Huebsch L. Progression of theumatoid arthritis following bone marrow transplantation arthritis following bone marrow transplantation

12 Faubes G. Do immunologists dream of electric mice? Science 1994; 256: 886-8.

13 Moreland L W, Heck L W, Sullivan W, Pratt P W Koopman $W$ J. New approaches to the therapy of autoimmune diseases: rheumatoid arthritis as a paradigm. Am f Med Sci 1993; 305: 40-51.

14 Elliott M J, Maini R N, Feldmann M, et al. Randomised double-blind comparison of chimeric monoclonal antibody to tumour necrosis factor alpha (cA2) versus placebo in rheumatoid arthritis. Lancet 1994; 344: 1105-10.

15 Trentham D E, Dynesius-Trentham R A, Orav E J, et al. Effects of oral administration of type 2 collagen on Effects of oral administration of type 2 collagen
rheumatoid arthritis. Science 1993; 261: 1727-30.
16 Lemmel E M, Brackertz D, Franke M, et al. Results of a multicenter placebo-controlled double blind randomized phase III clinical study of treatment of rheumatoid arthritis with recombinant interferon-gamma. Rheumatol Int 1988; 8: 87-93.

17 Cannon GW, Pincus S H, Emkey R D, et al. Double blind trial of recombinant interferon-gamma versus placebo in the treatment of rheumatoid arthritis. Arthritis Rheum 1989; 32: 964-73

18 Haagsma C J, van Riel P L C M, de Rooij A M, et al. Combination of methotrexate and sulphasalazine versus methotrexate alone: a randomized open clinical trial in rheumatoid arthritis patients resistan to sulphasalazine therapy. $\mathrm{Br} F$ Rheumatol 1994; 33: 1049-55.

19 Tugwell $\mathrm{P}$, Pincus T, Yocum D, et al. Combination therapy with cyclosporine and methotrexate in severe rheumatoid arthritis. NEngl f Med 1995; 333: 137-41.

20 O'Dell J, Hairs C, Erikson N, et al. Triple DMARD therapy for rheumatoid arthritis: efficacy [abstract]. Arthritis Rheum 1994; 37(suppl): S295. 